\title{
LncRNA Bmp1 promotes the healing of intestinal mucosal lesions via the miR-128-3p/PHF6/PI3K/AKT pathway
}

\author{
Mengmeng Zhuang ${ }^{1,2}$, Yuequ Deng ${ }^{1,2}$, Wenwen Zhang ${ }^{1,2}$, Bo Zhu ${ }^{1,2}$, Hao Yan ${ }^{1,2}$, Jiaqi Lou ${ }^{1,2}$, Pan Zhang ${ }^{1,2}$, \\ Qingwei Cui ${ }^{1,2}$, HaO Tang ${ }^{1,2}$, Han Sun ${ }^{1,2}$ and Yong Sun (1) ${ }^{1,2}$
}

\begin{abstract}
Intestinal mucosal injuries are directly or indirectly related to many common acute and chronic diseases. Long noncoding RNAs (IncRNAs) are expressed in many diseases, including intestinal mucosal injury. However, the relationship between IncRNAs and intestinal mucosal injury has not been determined. Here, we investigated the functions and mechanisms of action of IncRNA Bmp1 on damaged intestinal mucosa. We found that Bmp1 was increased in damaged intestinal mucosal tissue and Bmp1 overexpression was able to alleviate intestinal mucosal injury. Bmp1 overexpression was found to influence cell proliferation, colony formation, and migration in IEC-6 or HIEC-6 cells. Moreover, miR-128-3p was downregulated after Bmp1 overexpression, and upregulation of miR-128-3p reversed the effects of Bmp1 overexpression in IEC-6 cells. Phf6 was observed to be a target of miR-128-3p. Furthermore, PHF6 overexpression affected IEC-6 cells by activating PI3K/AKT signaling which was mediated by the miR-128-3p/PHF6 axis. In conclusion, Bmp1 was found to promote the expression of PHF6 through the sponge miR-128-3p, activating the PI3K/AKT signaling pathway to promote cell migration and proliferation.
\end{abstract}

\section{Introduction}

The intestinal mucosa functions physiologically as a defensive barrier, preventing bacteria and endotoxins (usually present in the gastrointestinal tract) from accessing extraintestinal tissues and organs. Maintaining the normal intestinal barrier function requires complex interactions of various defense mechanisms, including the ecological balance of the local intestinal flora, peristalsis, preservation of the mucus layer and epithelial cell barrier, and maintenance of normal epithelial cell turnover, immune function, and the enterohepatic axis. In addition, the loss of the intestinal barrier function appears to play a role in the development of systemic infections and/or multiple organ failure

\footnotetext{
Correspondence: Yong Sun (sunyong_97@163.com)

${ }^{1}$ Department of Burn Surgery, the Affiliated Huaihai Hospital of Xuzhou Medical University, Xuzhou 221004 Jiangsu Province, China

${ }^{2}$ Department of Burn Surgery, the 71st Group Army Hospital of PLA, Xuzhou, Jiangsu Province, China

These authors contributed equally: Mengmeng Zhuang, Yuequ Deng, Wenwen Zhang
}

Edited by R. Mantovani
(MOF) in some patients ${ }^{1}$. Therapeutic measures that help support the intestinal barrier function include maintaining an effective circulating blood volume, rapid identification and control of infection, the appropriate use of antibiotics, and optimal nutritional support. Despite the variety of strategies to restore the intestinal barrier function, effective treatment is currently limited. Therefore, elucidating the key molecular mechanisms mediating intestinal mucosal injury will facilitate treatment and improve prognosis.

RNA molecules that are not translated into proteins are called non-coding transcripts. These long non-coding RNAs (lncRNAs) represent a class of transcripts that are longer than 200 nucleotides and have no protein-coding capabilities $^{2,3}$. Recently, many lncRNAs related to the intestinal mucosa have been identified by highthroughput analysis of expression profiles. For example, downregulation of IncRNA ANRIL in the intestinal mucosa is associated with tumor risk, tumor activity, and increased levels of proinflammatory cytokines ${ }^{4}$. lncRNA

\section{(c) The Author(s) 2021}

(c) (i) Open Access This article is licensed under a Creative Commons Attribution 4.0 International License, which permits use, sharing, adaptation, distribution and reproduction cc) in any medium or format, as long as you give appropriate credit to the original author(s) and the source, provide a link to the Creative Commons license, and indicate if changes were made. The images or other third party material in this article are included in the article's Creative Commons license, unless indicated otherwise in a credit line to the material. If material is not included in the article's Creative Commons license and your intended use is not permitted by statutory regulation or exceeds the permitted use, you will need to obtain permission directly from the copyright holder. To view a copy of this license, visit http://creativecommons.org/licenses/by/4.0/. 
H19 can inhibit the expression of p53 protein, microRNA 34a, and let-7, and promote the proliferation and epithelial regeneration of intestinal epithelial cells (IECs) ${ }^{5}$. In our previous study, we found, using lncRNA microarray, that lncRNA Bmp1 expression was significantly increased in the intestines of burned mice. The length of mouse Bmp1 is 4464 bp and its gene is located on chromosome 14qD2 (https://www.genome.ucsc.edu/). It was first discovered in bone and reported to play multiple functions in bone formation. At present, there are few studies on Bmp1, and it is not clear whether Bmp1 can promote the repair of intestinal mucosa in burned mice.

Recent studies have shown that IncRNA can interact with miRNA to become a competitive endogenous RNA (ceRNA), thereby regulating the expression of target genes $^{6}$. MicroRNAs (miRNAs) play important roles in gene regulation by binding to the $3^{\prime}$-untranslated region ( $3^{\prime}$ UTR) of the target mRNA, which in turn leads to mRNA degradation or inhibition of translation ${ }^{7}$. For example, lncRNA-1604 inhibits miR-200c, resulting in Zinc finger E-box binding homeobox (ZEB) overexpression and promoting embryonic stem cell differentiation $^{8}$. LncRNA H19 regulates the expression of its target spaghetti via sponge miR-141 ${ }^{9}$. MiR-128-3p is a brain-rich microRNA that is highly enriched and upregulated in the embryonic mouse brain. It is thought to be involved in the control of neurogenesis and synaptogenesis. It has been reported that miR-128 regulates neuronal migration and growth through the gene $P h f 6^{10}$. We found in the pre-experiment that Bmp1 contained two binding sites of miR-128-3p. However, it is not clear whether Bmp1 can promote proliferation and migration through sponge adsorption miR-128-3p.

Plant homeodomain finger protein 6 (PHF6), located on Xq26-q27, was initially reported as a single gene mutation in Börjeson-Forssman-Lehmann syndrome, which is characterized by X-chromosome-related intellectual disabilities $^{11}$. PHF6 encodes a plant homology domain (PHD) factor containing four nuclear localization signals and two incomplete PHD zinc finger domains ${ }^{12}$. Studies have shown that PHF6 may significantly promote proliferation and migration and the epithelial-mesenchymal transition (EMT) in hepatocellular carcinoma (HCC) cells $^{13}$. PHF6 might play a crucial role in mouse neuronal migration during the early phases of development in primary cortical neurons and the developing mouse brain $^{14}$. It is reported that the $3^{\prime} \mathrm{UTR}$ of the Phf6 gene contains a sequence complementary to the seed region of miR-128-3p, suggesting that miR-128-3p can directly target $P h f 6^{10}$. Therefore, we speculate that miR-128-3p may play a physiological role through PHF6.

The PI3K/AKT pathway plays a role in a variety of physiological activities, and its activation is closely related to cell proliferation, survival, migration, invasion, metastasis, and tumor growth ${ }^{15}$. The activation of the PI3K/Akt pathway can enhance the wound healing response, increase the expression of EMT markers (MMP 9), and decrease the expression of cell adhesion/epithelial markers (ZO-1, E-cadherin) ${ }^{16}$. Matrix metalloproteinases (MMPs) can degrade all components of the extracellular matrix (ECM) and play key roles in normal physiological processes involving ECM remodeling, such as wound healing, angiogenesis, and development. MMP-9 has been shown to be involved in the migration of several cell types, namely macrophages, $\mathrm{T}$ lymphocytes, and eosinophils, through the recombinant basement membrane ${ }^{17}$. Zonula occludens $(\mathrm{ZO})-1$ is a multi-domain scaffold protein, which is known to play a key role in the targeting, anchoring, and aggregation of cross-linked cell adhesion molecules and cytoskeleton proteins, as well as in the establishment of cell-cell adhesion and the maintenance of stable tissue structure ${ }^{18}$. E-cadherin plays an important role in cell-cell adhesion, epithelial-to-mesenchymal transformation, cell migration, and invasion ${ }^{19}$.

In this study, we explore the effects of Bmp1 on cell proliferation and migration by inducing overexpression of Bmp1. We have also investigated the associated mechanism to determine whether Bmp1 works through a competing endogenous RNA (ceRNA) mechanism to reduce miR-128-3p bioavailability and induce the transcription of the miR-128-3p target gene Phf6, which significantly increases the phosphorylation levels of PI3K and AKT. Our results provide a reference for further analysis of the IncRNA-miRNA-mRNA-signaling pathway network during intestinal mucosal injury repair.

\section{Results}

Bmp1 expression increases in the burned mouse model

To detect changes in Bmp1 expression, intestinal mucosal RNA was extracted from burned mice. qRT-PCR results showed that the expression level of Bmp1 in the intestine of mice $24 \mathrm{~h}$ after burn was significantly higher than that in the control group (Fig. 1A). ELISA results showed that the levels of serum LPS, IL-6, and TNF- $\alpha$ in the burn group were significantly higher than those in the control group (Fig. 1B). Western blot results showed that the expression level of the migration-related protein MMP9 in the intestines of mice $24 \mathrm{~h}$ after burn was significantly lower than that in the control group, while the expression levels of E-cadherin, and ZO-1 were slightly lower than those in the control group (Fig. 1C).

\section{Bmp1 upregulation promotes cell proliferation and migration}

To clarify the role of Bmp1 in the repair of damaged intestinal mucosa, intestinal epithelial cells were cultured to mimic the intestinal mucosal barrier. The expression of Bmp1 in IEC- 6 or HIEC- 6 was altered by the transfection 
A

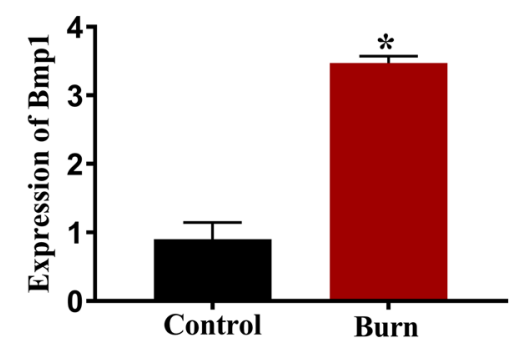

B
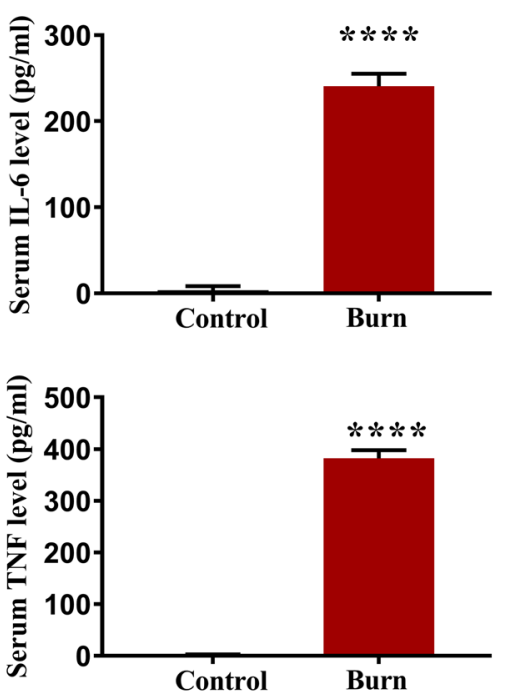

C

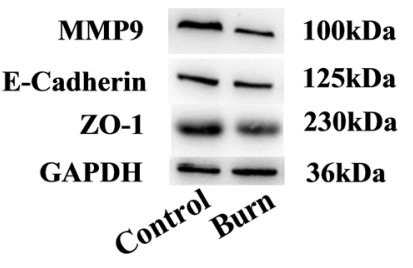

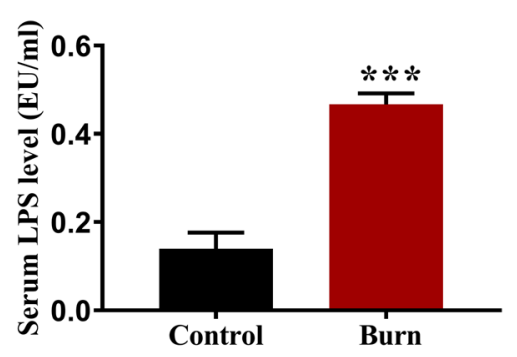

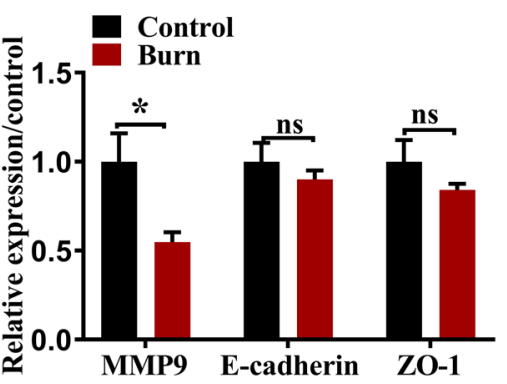

Fig. 1 Expression of Bmp 1, TNF-a, IL-6, LPS, MMP-9, E- cadherin, and ZO-1 in intestinal tissue of burned mice. A Expression of Bmp1 ( $n=10$ ), B TNF-a, IL-6, LPS $(n=10)$, C cell migration-related protein MMP9, E-cadherin, and ZO-1 were detected by qRT-PCR, ELISA, and western blot, respectively $(n=10)$. All data were demonstrated as mean \pm standard deviation (SD) from three independent experiments performed in duplicate. ${ }^{*} p<.05,{ }^{* *} p<.001$, and ${ }^{* * * *} p<.0001$ were all significant differences.

of adenovirus. The effect of the transfection on Bmp1 expression was confirmed by qRT-PCR analysis (Fig. 2A). The CCK-8 assay was performed to monitor cell proliferation and the result showed that the proliferation of Bmp1-knockdown IEC-6 or HIEC-6 cells was significantly decreased, while overexpression of Bmp1 could significantly promote the proliferation of cells (Fig. 2B). Furthermore, the colonies remarkably increased in IEC-6 or HIEC-6 cells after Bmp1 transfection, while decreased significantly after si-Bmp1 transfection (Fig. 2C). Woundhealing assays verified the relationship between Bmp1 and cell migration in IEC-6 or HIEC-6 cells. The results showed that Bmp1 can promote cell migration, while knockdown of Bmp1 inhibits cell migration (Fig. 2D). The western blot results showed that the MMP9 content in Bmp1-knockdown cells decreased significantly, while the contents of E-cadherin and ZO-1 increased significantly. On the other hand, MMP9 expression increased and the expression of E-cadherin and $\mathrm{ZO}-1$ decreased in the Bmp1 overexpression group compared with the control group (Fig. 2E). The results of the immunofluorescence assay showed that the E-cadherin content in Bmp1 


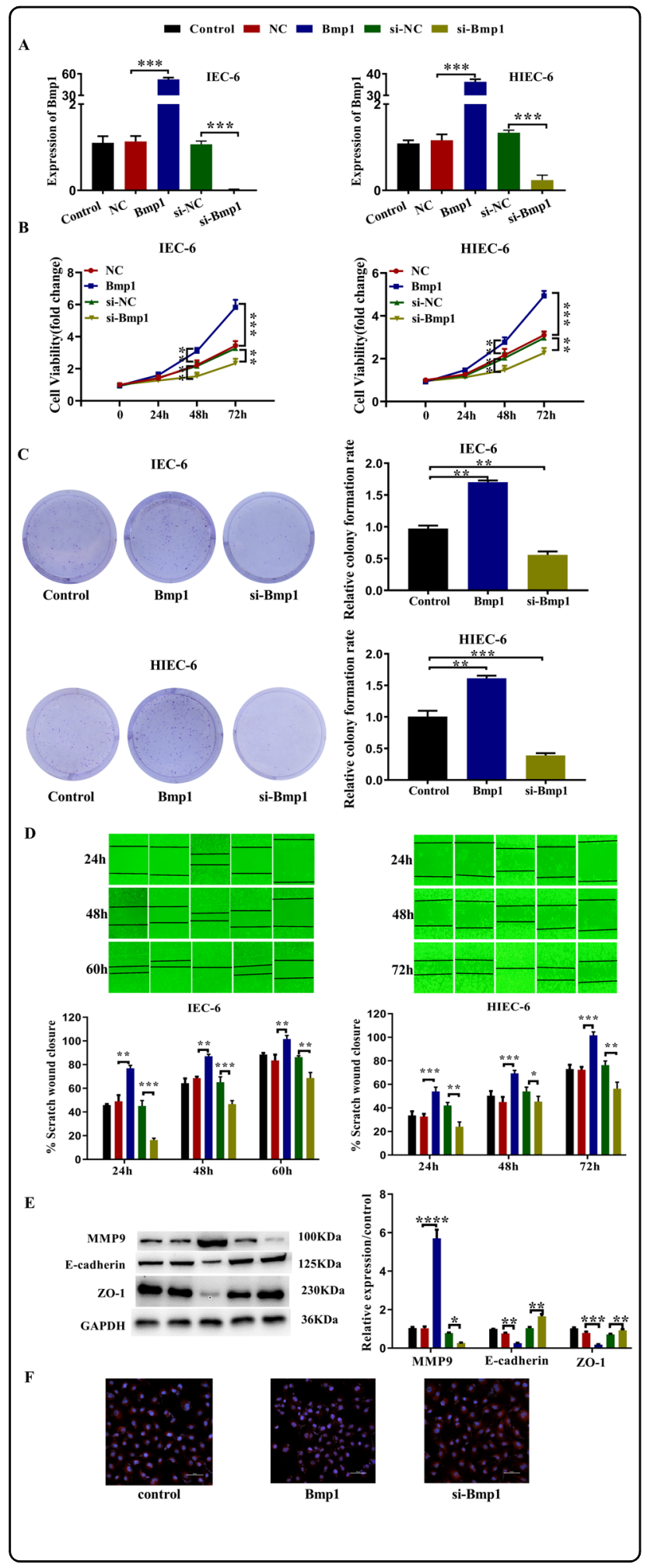

knockdown cells increased significantly, while the content of E-cadherin decreased significantly in the Bmp1 group compared with the control group (Fig. 2F).
Fig. 2 Overexpression of Bmp1 promotes the migration and proliferation of IEC- 6 cells. A The alter expression of Bmp1 was determined using qRT-PCR $(n=3)$. B Cell viability $(n=3)$, C colony formation assays were performed to exam the function of Bmp1 in cells proliferation. D Cell migration ability was quantified by the percentage of the area of cells migrated to the wound in the total wound area $(n=3)$. E MMP9, E-cadherin, and ZO-1 were detected by western blot $(n=3)$. F Representative immunofluorescence analysis showed the content of E-cadherin in treated IEC- 6 cells $(n=3)$. All data were demonstrated as mean \pm SD from three independent experiments performed in duplicate. ${ }^{*} p<0.05,{ }^{* *} p<0.01,{ }^{* * *} p<0.001$, and ${ }^{* * * *} p<0.0001$ were all significant differences.

\section{Bmp1 has a protective effect on the intestinal tract of burned mice}

To identify the underlying effects of Bmp1 on the burned mice, RNA samples were collected for detecting expression changes in Bmp1.

Firstly, we established the third-degree mouse burn model (Fig. 3A) and verified the expression levels of Bmp1 in each group by qRT-PCR (Fig. 3B). Histological examination of H\&E-stained sections under light microscopy showed that the intestinal mucosal injury in the Burn + Bmp1 group was slighter than that in the burn group (Fig. 3C, Table S1, and Table S2). The results of the ELISA showed that the levels of serum LPS, IL-6, and TNF- $\alpha$ in the burn group were significantly higher than those in the control group while the levels in the Burn $+B m p 1$ group were intermediate between the two groups (Fig. 3D). The western blot results showed that, compared with the control group, the expression of MMP9 in the Burn + Bmpl group increased significantly, while the expression of E-cadherin and ZO-1 decreased significantly (Fig. 3E).

\section{Bmp1 serves as a miRNA sponge for miR-128-3p}

Studies have shown that lncRNAs can be used as ceRNAs to sponge miRNAs, leading to the de-expression of miRNA targets. To investigate the mechanism underlying Bmpl's role in cell proliferation and migration, we used bioinformatic analysis to predict the potential miRNAs that may bind to Bmp1. Analysis with miRWalk software showed that miR-128-3p was predicted to bind to Bmp1 (Fig. 4A). qRT-PCR results proved that Bmp1 negatively regulated the expression of miR-128-3p (Fig. 4B). To further determine whether Bmp1 directly regulates miR-128-3p, we cloned the hypothetical miR-128-3p target-binding sequence into a luciferase construct. The miR-128-3p mimic and Bmp1 wild-type or mutant reporter genes were transfected into $293 \mathrm{~T}$ cells (Fig. 4C). It was found that the miR-128-3p mimic significantly reduced luciferase activity of the $\mathrm{Bmp} 1$ wild-type reporter gene, and the mutation of the Bmp1 target successfully 


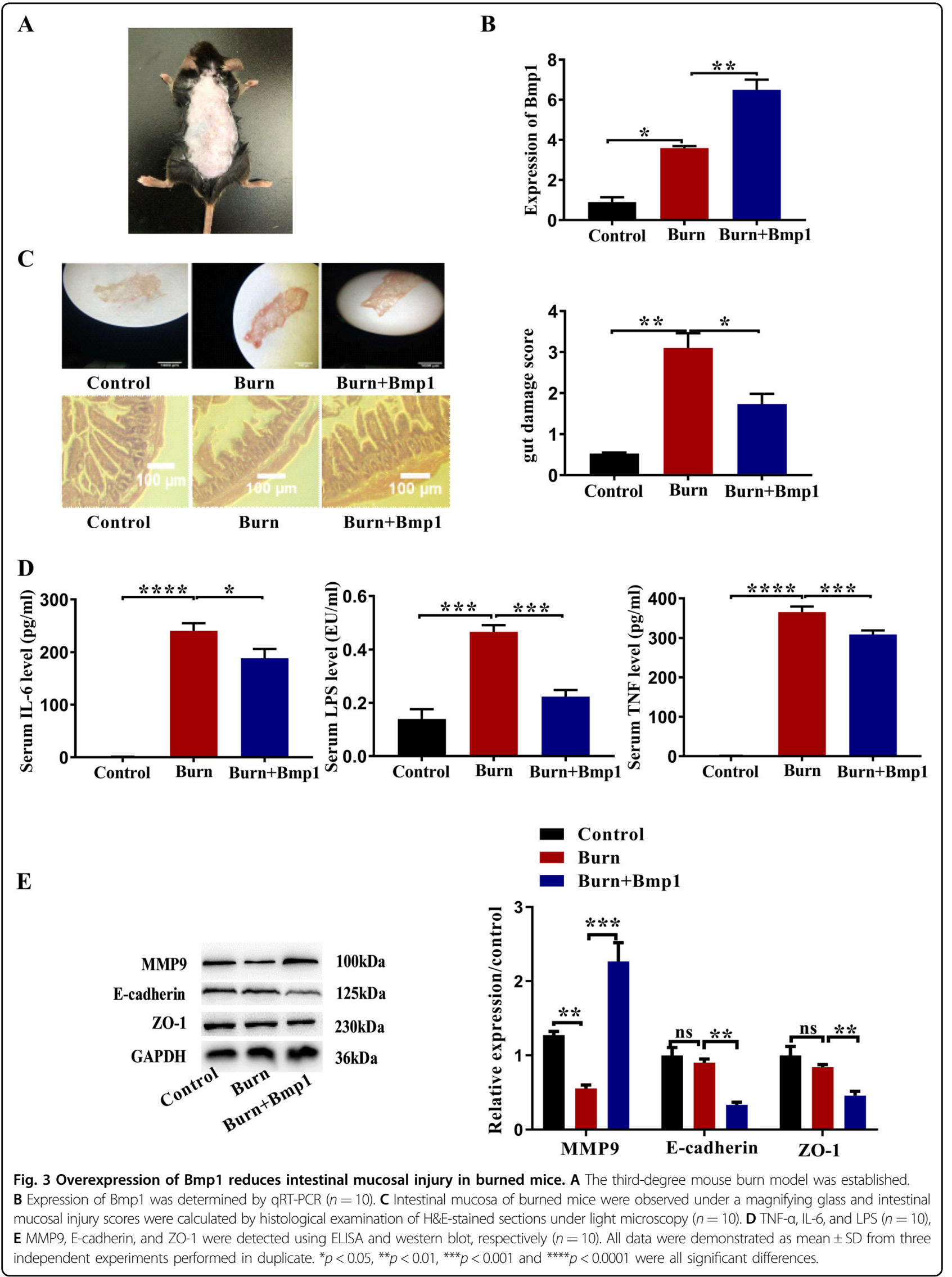


A

\begin{tabular}{|c|c|c|c|c|c|c|c|c|c|}
\hline Mirna $\triangle$ & Refseqid $\triangle$ & Genesymbol $\Delta$ & Duplex $\triangle$ & $\begin{array}{l}\text { Binding } \\
\text { P-Value }\end{array}$ & Position $\triangle$ & $\begin{array}{l}\text { Binding } \\
\text { Site } \triangle\end{array}$ & $\mathrm{Au} \triangle \Delta$ & Me $\triangle$ & $\begin{array}{l}\mathrm{N} \\
\text { Pairings } \triangle\end{array}$ \\
\hline $\begin{array}{l}\text { mmu- } \\
\text { miR- } \\
128-3 p\end{array}$ & NM_009755 & Bmp1 & $(((((. . .(((((((((((\ldots \&)))))))))))))))))$. & 1.00 & CDS & 1415,1432 & 0.34 & -7.382 & 16 \\
\hline $\begin{array}{l}\text { mmu- } \\
\text { miR- } \\
128-3 p\end{array}$ & NM_009755 & Bmp1 & 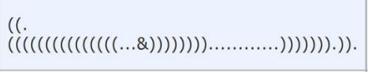 & 0.85 & 3UTR & 3392,3423 & 0.4 & -5.495 & 17 \\
\hline $\begin{array}{l}\text { mmu- } \\
\text { miR- } \\
128-3 p\end{array}$ & NM_009755 & Bmp1 & $(((((((\ldots . .((((((((((\&)))))))))))))))))))$. & 0.92 & CDS & 2974,2992 & 0.5 & -11.375 & 17 \\
\hline
\end{tabular}

B
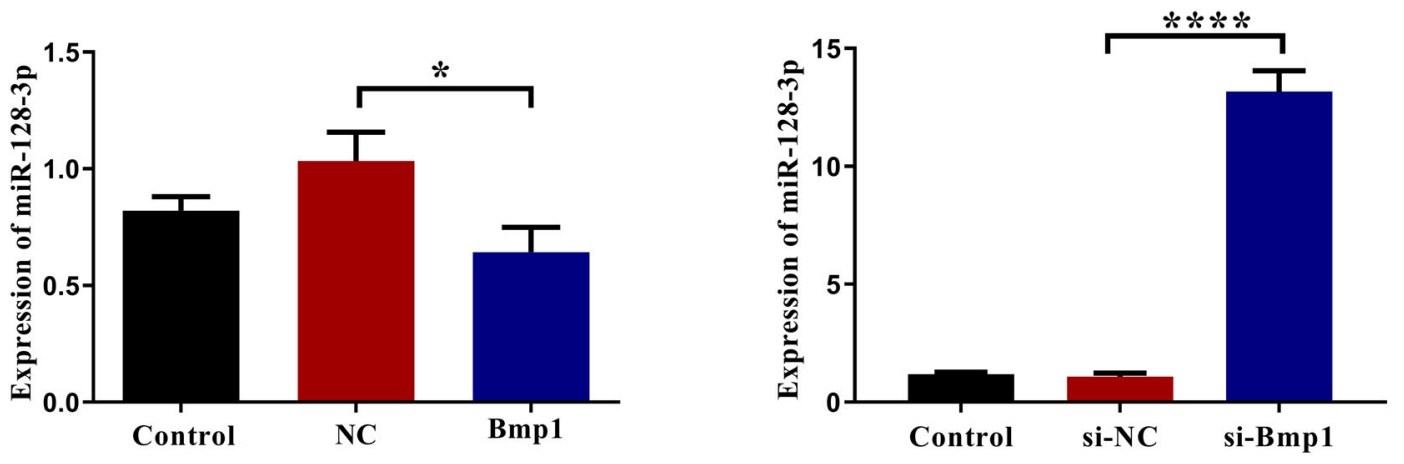

C

miR-128-3p

3' ... UUUCUCUGGCCAAGUGACACU...5'

| | | | | |

Bmp1-wt1

5'... ACAUGCACUGUGU... 3'

Bmp1-mut1

5'... ACAUGAAGUCUCU... 3'

miR-128-3p

Bmp1-wt2

Bmp1-mut2

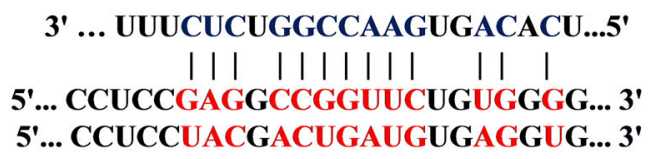

D

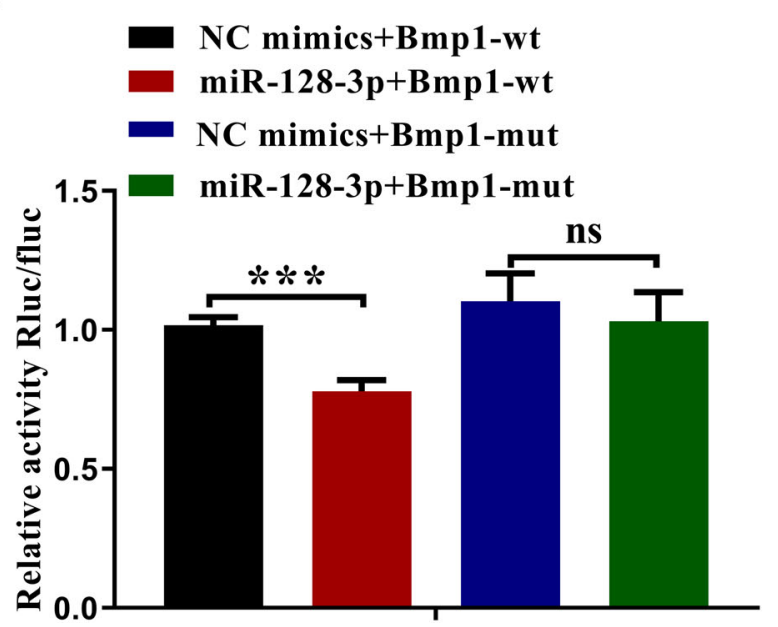

Fig. 4 Bmp1 functions as an endogenous sponge of miR-128-3p. A The predicted sequence between Bmp1 and miR-128-3p using miRWalk. B Expression of miR-128-3p was determined using qRT-PCR $(n=3)$. C A schematic drawing indicated the mutant binding sites of miR-128-3p with respect to Bmp1. D Luciferase reporter assay was performed in 293T cells cotransfected with miR-128-3p mimic or mimic NC and luciferase reporters constructed with Bmp1-wt or Bmp1-mut. The luciferase activity was normalized to the ratio of firefly to the renilla activity $(n=3)$. All data were demonstrated as mean \pm SD from three independent experiments performed in duplicate. ${ }^{*} p<0.05,{ }^{* *} p<0.01,{ }^{* * *} p<0.001$ and, ${ }^{* * * *} p<0.0001$ were all significant differences. 
reversed the previous inhibition (Fig. 4D). These findings indicate the binding conditions of miR-128-3p and Bmp1.

\section{Upregulation of miR-128-3p alters the impact of Bmp1 overexpression on IEC- 6 cells}

To further investigate how miR-128-3p was involved in Bmp1-mediated cell proliferation and migration, rescue assays were performed. As shown in Fig. 5A, the miR-128$3 \mathrm{p}$ expression was upregulated or suppressed after transfection with miR-128-3p mimic or miR-128-3p inhibitor, respectively. The CCK- 8 results showed that overexpression of Bmp1 and knockdown of miR-128-3p could significantly promote cell proliferation, while knockdown of Bmp1 and overexpression of miR-128-3p could significantly inhibit cell proliferation. When Bmp1 and miR-128-3p were overexpressed or knocked down at the same time, miR-128-3p could reverse the effects of Bmp1 on cell proliferation (Fig. 5B). The wound-healing assay and western blot results showed that overexpression of Bmp1 and knockdown of miR-128-3p promoted both cell migration and the expression of MMP9, and reduced the expression of E-cadherin and ZO-1. After knocking down Bmp1 and over-expressing miR-128-3p, IEC-6 cell migration was inhibited, MMP9 concentrations were significantly reduced and the expression of E-cadherin and ZO-1 were significantly increased. When Bmp1 and miR-128-3p were overexpressed or knocked down at the same time, miR-128-3p reduced the effect of Bmp1 on cell migration and migration-related proteins MMP9, E-cadherin, and ZO-1 (Fig. 5C, D). Immunofluorescence assay results showed that overexpression of Bmp1 and knockdown of miR-128-3p significantly reduced the expression of E-cadherin, while E-cadherin levels increased on Bmp1 knockdown and miR-128-3p overexpression. When Bmp1 and miR-128-3p were overexpressed or knocked down at the same time, miR-128-3p reduced the effect of Bmp1 on E-cadherin (Fig. 5E).

\section{Phf6 is targeted by miR-128-3p}

Because lncRNA, as competitive ceRNAs, compete with mRNA for miRNA binding ${ }^{20}$, we searched four bioinformatics tools (miRDB, PicTar, TargetScan, and RNAInter) and jointly predicted that 11 genes may be biological targets of miR-128-3p (Fig. 6A). Through qRT-PCR, we identified Phf6 as our research object (Fig. 6B). We performed a bioinformatic analysis using TargetScan and identified the potential binding sites between miR-128-3p and Phf6 (Fig. 6C). To verify whether Phf6 is indeed targeted by miR-128-3p, we further constructed the reporter gene vectors Phf6-3' UTR-wild and Phf6-3' UTR-mut (Fig. 6D). After briefly co-transfecting 293T cells with miR-128-3p mimics/NC mimic and the reporter gene vector Phf6-3'UTR-wild/mut, the cells were assayed for dual-luciferase reporter genes. In the Phf6-3' UTR-wild transfection group, treatment with the miR-128-3p mimic reduced the relative activity of luciferase while this did not occur in the group transfected with Phf6-3'UTR-mut (Fig. 6E). This indicates that miR-128-3p reduces Phf6 expression by binding to Phf6 3'UTR. qRT-PCR showed that the miR-128-3p mimic decreased Phf6 levels significantly while miR-128-3p inhibitors significantly increased Phf6 levels (Fig. 6F). Western blotting also confirmed that miR-128-3p inhibited the expression of PHF6 protein in IEC-6 cells (Fig. 6G). These results confirm that Phf6 is a direct target of miR-128-3p.

\section{PHF6 rescues the effect of miR-128-3p}

To further investigate the involvement of PHF6 in miR128-3p-mediated cell proliferation and migration, we performed rescue assays. The CCK- 8 results showed that overexpression of PHF6 and knockdown of miR-128-3p significantly promoted cell proliferation while knockdown of PHF6 and overexpression of miR-128-3p inhibited proliferation. When miR-128-3p and PHF6 were overexpressed or knocked down at the same time, PHF6 reduced the effect of miR-128-3p on cell proliferation (Fig. 7A). Wound-healing assay and western blot results showed that overexpression of PHF6 and knockdown of miR-128-3p could significantly promote cell migration, promote the expression of MMP9, and significantly reduce the expression of E-cadherin and ZO-1. Knockdown of PHF6 and overexpression of miR-128-3p inhibited both cell migration and MMP9 expression, while increasing the expression of E-cadherin and ZO-1. When miR-128-3p and PHF6 were overexpressed or knocked down at the same time, PHF6 weakened the effect of miR-128-3p on cell migration and the migration-related proteins MMP9, E-cadherin, and ZO-1(Fig. 7B, C). Immunofluorescence assay results showed that overexpression of PHF6 and knockdown of miR-128-3p could significantly reduce the expression of E-cadherin. Knockdown of PHF6 and overexpression of miR-128-3p significantly increased the expression of E-cadherin. When miR-128-3p and PHF6 were overexpressed or knocked down at the same time, PHF6 reduced the effect of miR-128-3p on E-cadherin (Fig. 7D).

\section{PHF6 plays a physiological role in IEC- 6 cells possibly by activating PI3K/AKT signaling}

Western blot results showed that overexpression of Bmp1 and PHF6 could activate the PI3K/AKT signaling pathway, while knockdown of Bmp1 and PHF6 inhibited the pathway. When Bmp1 and miR-128-3p were overexpressed or knocked down at the same time, miR-128-3p reduced the effect of Bmp1 on PI3K/AKT activation. When Bmp1, miR-128-3p, and PHF6 were overexpressed or knocked down at the same time, PHF6 reduced the effect of miR-128-3p and Bmp1 on the pathway. 


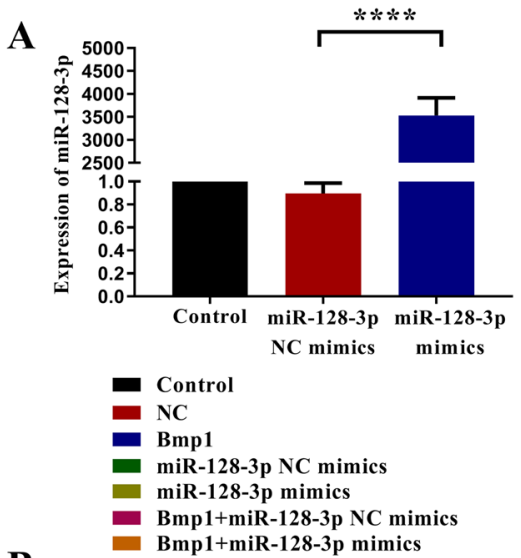

B

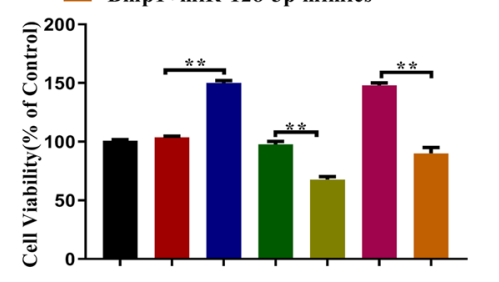

C
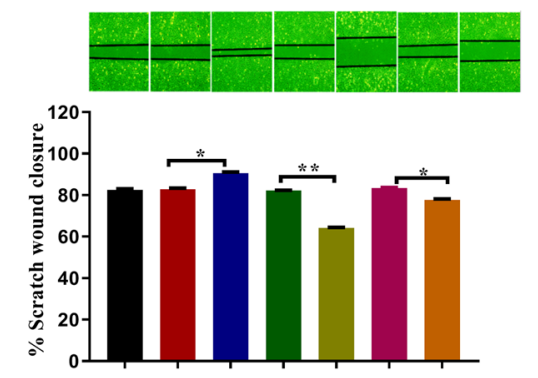

D
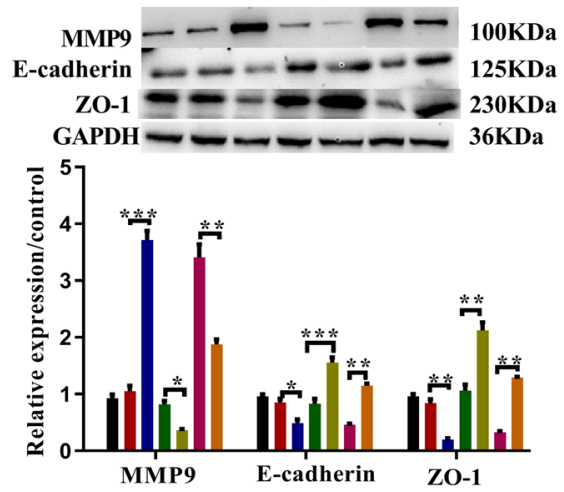

$\mathbf{E}$

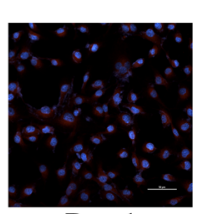

Bmp1

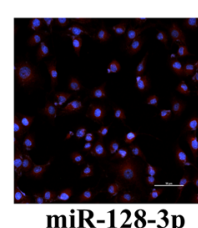

mimics

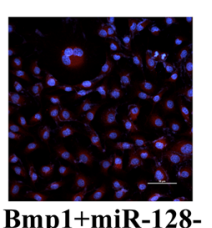

$3 p$ mimics

Fig. 5 MiR-128-3p mimic can rescue the impacts of overexpression of bmp1 on IEC-6 cells. A The expression of miR-128-3p in IEC-6 cells transfected with miR-128-3p mimic or miR-128-3p inhibitor were determined using qRT-PCR $(n=3)$. B Cell viability $(n=3)$, C cell migration ability $(n=3)$, D E-cadherin and ZO-1 were analyzed using cell counting kit-8 assay, wound healing assay and western blot, respectively $(n=3)$.

E Representative immunofluorescence analysis showed the content of E-cadherin in treated IEC-6 cells $(n=3)$. All data were demonstrated as mean \pm SD from three independent experiments performed in duplicate. ${ }^{*} p<.05,{ }^{* *} p<.01,{ }^{* * *} p<.001$ and ${ }^{* * *} p<.0001$ were all significant differences. 
A

C

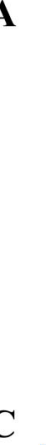

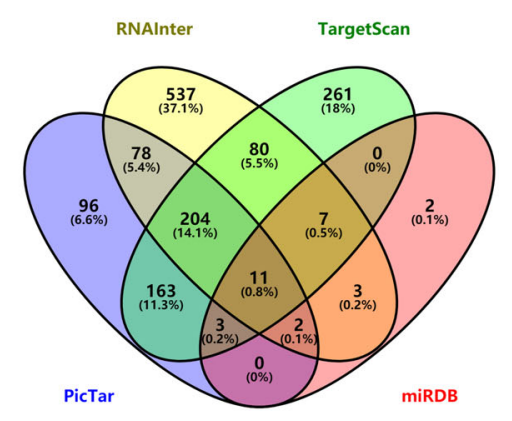

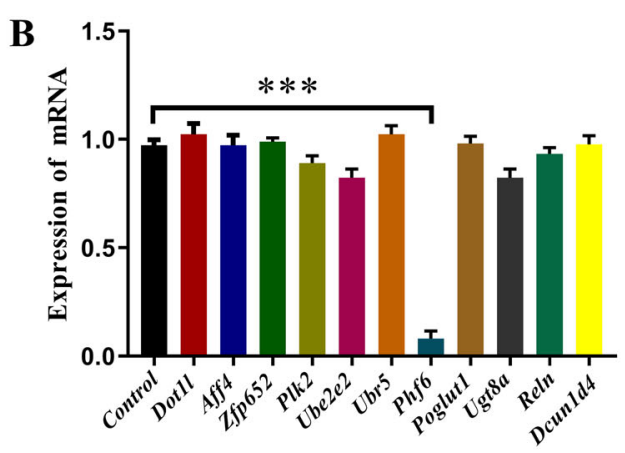

\begin{tabular}{|c|c|c|c|c|c|c|c|c|}
\hline & $\begin{array}{l}\text { Predicted consequential pairing of targe } \\
\text { miRNA (bottom) }\end{array}$ & egion (top) and & $\begin{array}{l}\text { Site } \\
\text { type }\end{array}$ & $\begin{array}{l}\text { Context++ } \\
\text { score }\end{array}$ & $\begin{array}{l}\text { Context++ score } \\
\text { percentile }\end{array}$ & $\begin{array}{c}\text { Weighted } \\
\text { context++ score }\end{array}$ & $\begin{array}{l}\text { Conserved branch } \\
\text { length }\end{array}$ & $\mathbf{P}_{\text {CT }}$ \\
\hline $\begin{array}{l}\text { Position 1322-1328 of Phf6 3' UTR : } \\
\text { mmu-miR-128-3p }\end{array}$ & $\begin{array}{l}\text { ACAGGUGGAAAALUACACUGLGC... } \\
\text { UULCUCUGGCCAAGUGACACU }\end{array}$ & 1111\|\| & $\begin{array}{c}7 \mathrm{mer}- \\
\mathrm{m} 8\end{array}$ & -0.17 & 88 & -0.17 & 2.288 & 0.24 \\
\hline $\begin{array}{l}\text { Position } 1672-1678 \text { of Phf6 } 3^{\prime} \text { UTR : } \\
\text { mmu-miR-128-3p }\end{array}$ & $\begin{array}{l}\text {... UULAAAUCUULAAAUCACUGLGU... } \\
\text { UUUCUCUGGCCAAGIGACACU }\end{array}$ & 1111111 & $\begin{array}{c}7 \mathrm{mer}- \\
\mathrm{m} 8\end{array}$ & -0.23 & 93 & -0.22 & 2.039 & 0.17 \\
\hline $\begin{array}{l}\text { Position 1979-1985 of Phf6 3' UTR } \\
\text { mmu-miR-128-3p }\end{array}$ & $\begin{array}{l}\text {... ACUACCUGUULUCUCACUGUGG. . } \\
\text { UUUCLCUGGCCAAGLGACAC }\end{array}$ & 1111111 & $\begin{array}{c}7 \mathrm{mer}- \\
\mathrm{m} 8\end{array}$ & -0.12 & 80 & -0.11 & 2.039 & 0.17 \\
\hline
\end{tabular}

D
miR-128-3p
3'....UUUCUCUGGCCAAGUGACACU... 5'
|||||| $\mid$
Phf6-3'UTR-wt 5'....CAGGUGGAAAAUUACACUGUGCU.... 3'
Phf6-3'UTR-mut1 5'....CAGGUGGAAAAUUAGAGUCUCCU.... 3'
miR-128-3p
$3^{\prime}$....UUUCUCUGGCCAAGUGACACU... $5^{\prime}$
|| || ||
Phf6-3'UTR-wt
5'....UUAAAUCUUUAAAUCACUGUGUC.... 3 '
Phf6-3'UTR-mut2
5 '....UUAAAUCUUUAAAUGAGUCUCUC.... $3^{\prime}$
miR-128-3p
Phf6-3'UTR-wt
3'....UUUCUCUGGCCAAGUGACACU... 5'
|||||| $\mid$
Phf6-3'UTR-mut3
5'....UACCUGUUUUUCUCACUGUGGU.... $3^{\prime}$
5'....UACCUGUUUUUCUGAGUCUCGU... 3'

$\mathbf{E}$

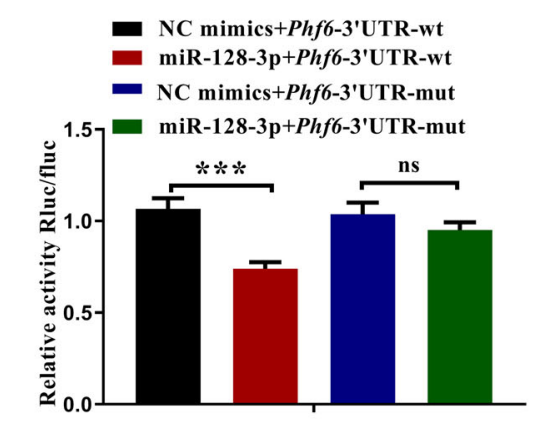

$\mathbf{F}$
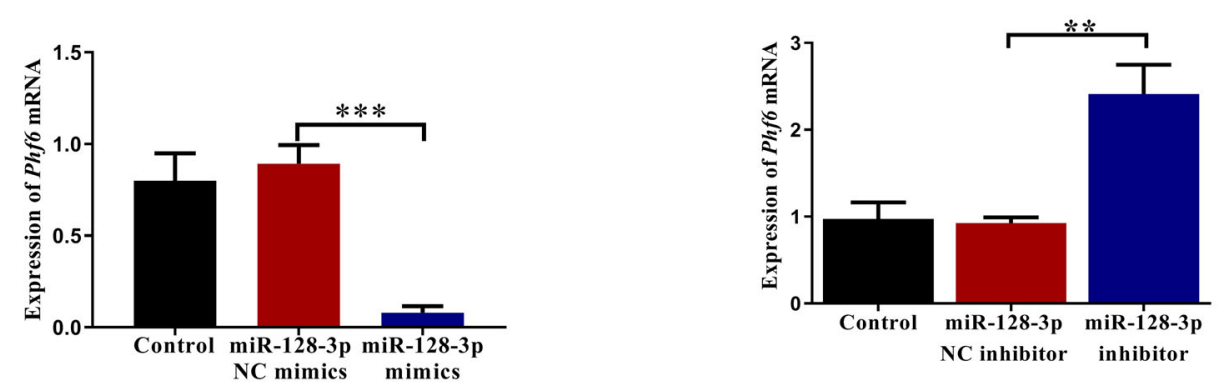

G
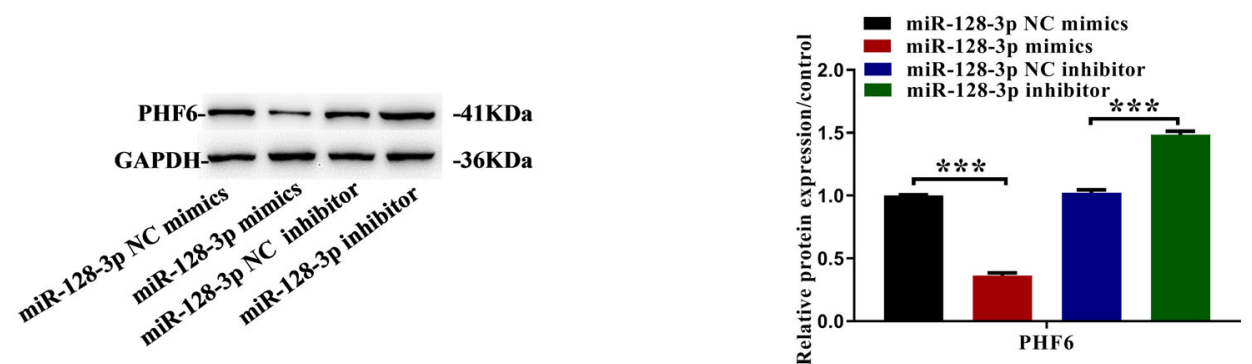

Fig. 6 Phf6 is the target gene of miR-128-3p. A A Venn diagram indicated that 30 genes may be functional targets of miR-128-3p. B qRT-PCR showed that miR-128-3p dramatically reduced the mRNA expression level of Phf6. C The predicted sequence between miR-128-3p and Phf6 using TargetScanMouse. D The mutant binding sites of miR-128-3p at the $3^{\prime}$ UTR of Phf6 mRNA. E Relative luciferase activities of Phf6-3' UTR-wt or Phf6-3' UTR-mut were analyzed in HEK293T cells after co-transfection with miR-128-3p mimic or mimic NC. The luciferase activity was normalized to the ratio of firefly to the renilla activity $(n=3)$. F, G qRT-PCR and western blot analysis were used to detect the PHF6 expression in IEC-6 cells transfected with miR-128-3p mimic and miR-128-3p inhibitor $(n=3)$. All data were demonstrated as mean \pm SD from three independent experiments performed in duplicate. ${ }^{*} p<0.05,{ }^{* *} p<0.01,{ }^{* *} p<0.001$, and ${ }^{* * *} p<0.0001$ were all significant differences. 


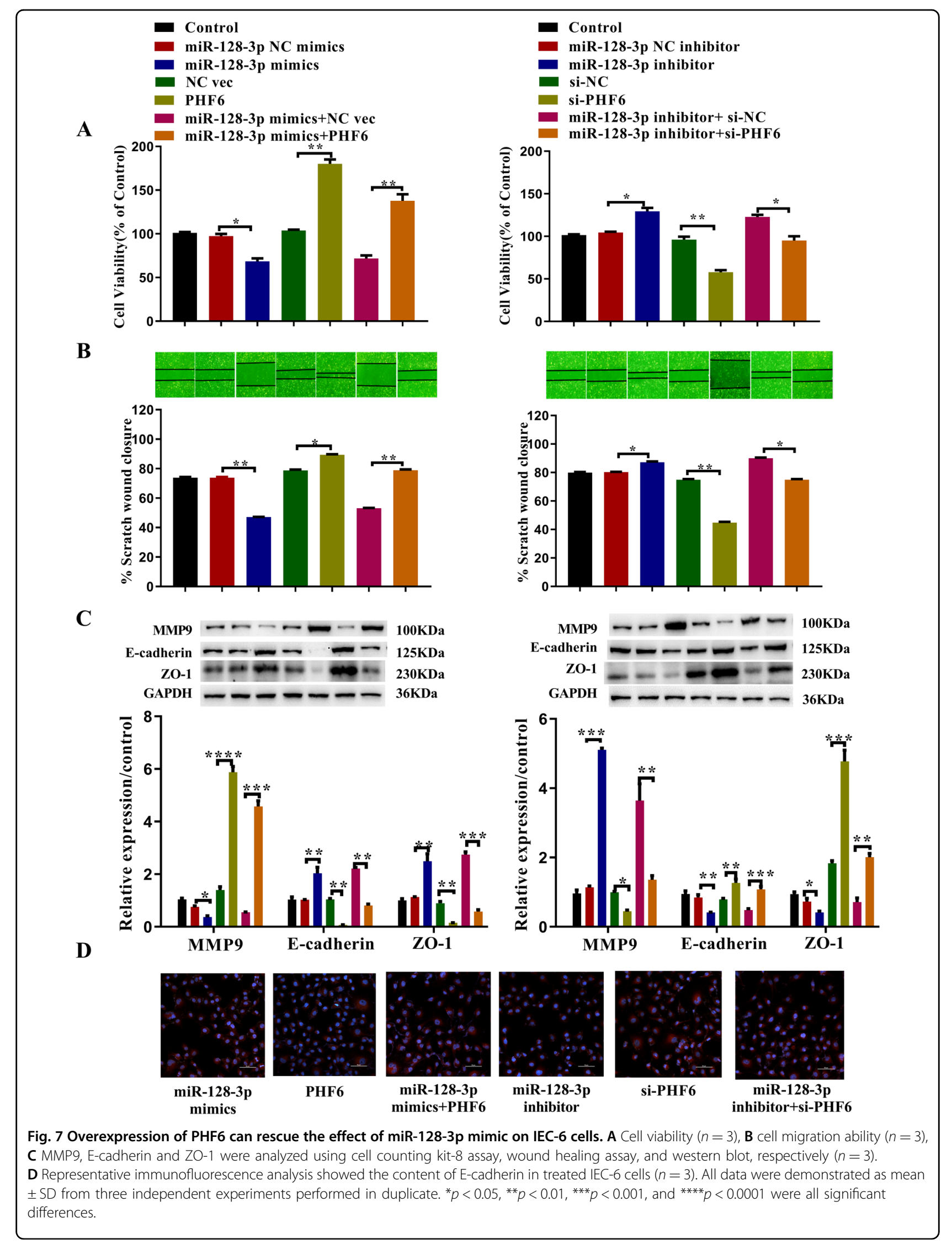


Interference with $\mathrm{AKT}$ expression altered the effects of PHF6 (Fig. 8A). The CCK-8 results showed that overexpression of Bmp1 and PHF6 promoted cell proliferation, while knockdown of both inhibited proliferation. At the same time, when Bmp1 and miR-128-3p were overexpressed or knocked down, miR-128-3p reduced Bmpl's promotion of cell proliferation. When Bmp1, miR-128-3p, and PHF6 were overexpressed or knocked down at the same time, PHF6 reduced the effects of both miR-128-3p and Bmp1 on cell proliferation. Interference with AKT expression altered PHF6's effects on proliferation (Fig. $8 \mathrm{~B})$. The wound-healing assay showed that overexpression of Bmp1 and PHF6 could significantly promote cell migration, while knockdown of Bmp1 and PHF6 reversed this effect. However, when Bmp1 and miR-128$3 p$ were overexpressed or knocked down, miR-128-3p could reverse the effects of Bmp1 on cell migration. When Bmp1, miR-128-3p, and PHF6 were overexpressed or knocked down at the same time, PHF6 could weaken the effects of miR-128-3p and Bmp1 on cell migration. Interference with the AKT expression level altered the effects of PHF6 on cell migration (Fig. 8C).

\section{Discussion}

Burns can change the structure and function of the intestinal tract and damage the intestinal mucosal barrier, resulting in intestinal bacterial translocation and severe burn infections $\mathrm{s}^{21,22}$. Therefore, protecting the integrity of the intestinal structure after burns and promoting the recovery of the intestinal mucosa have important clinical significance. This study shows that the content of Bmp1 is upregulated after intestinal mucosal injury. The overexpression of Bmp1 has a protective effect on the intestine of burned mice and can significantly improve the proliferation and migration ability of IEC- 6 or HIEC- 6 cells through the lncRNA Bmp1/miR-128-3p/PHF6/PI3K/ AKT pathway.

LncRNAs perform important functions in the regulation of many biological processes, including gene transcription and translation. Abnormal expression of lncRNAs occurs in many diseases. In this study, we found that the expression level of Bmp1 in the mouse intestine increased significantly at $24 \mathrm{~h}$ after the burn, while the intestinal mucosal injury was severe, and the expression level of the migration-related protein MMP9 was significantly decreased. To verify the physiological role of Bmp1, we used IEC-6 or HIEC- 6 cells to build an intestinal mucosal injury model in vitro. The results showed that Bmp1 could significantly promote the proliferation and migration of the cells, as well as the expression level of migration-related proteins such as MMP9. In addition, we also verified the role of Bmp1 in the burn mouse model. The intestinal mucosal injury of burned mice was significantly alleviated after intragastric injection of Bmp1, and the injury index was significantly lower than that of the burn group.

Although Bmp1 has been shown to be involved in the repair of damaged intestinal mucosa, the exact molecular mechanism is unclear. Recently, the "ceRNA" hypothesis has been proposed: that lncRNA functions by acting as a sponge for miRNA. Binding between lncRNAs and miRNAs blocks the inhibitory effects of miRNAs on mRNA ${ }^{23}$. For example, the long-chain non-coding RNA LNCSEMT regulates IGF2 expression through the sponge miR-125b, which promotes the development and growth of sheep muscle ${ }^{24}$. LncRNA lnc-HC regulates PPAR $\gamma$ mediated lipid metabolism through miR-130b-3p ${ }^{25}$. To explore the mechanism of intestinal mucosal repair mediated by Bmp1, bioinformatics analysis was performed and the results showed that miR-128-3p can bind to Bmp1. Then, we further explored the effect of miR-128$3 p$ on the proliferation and migration of IEC- 6 cells and the relationship between miR-128-3p and Bmp1. First, our results verified that miR-128-3p negatively regulates the proliferation and migration of IEC- 6 cells: miR-128-3p overexpression downregulates the expression of MMP9 and inhibits cell migration, whereas the miR-128-3p inhibitor upregulates MMP9 and promotes cell migration. Second, Bmp1 negatively regulates the expression of miR-128-3p: overexpression of Bmp1 reduces the expression of miR-128-3p and inhibits Bmp1 upregulation of miR-128-3p expression. To determine whether there is direct binding between Bmp1 and miR-128-3p, we performed a luciferase reporter gene assay which showed that miR-128-3p inhibits the luciferase activity of the vector containing the Bmp1 sequence. Mutation of the hypothetical miR-128-3p target site reversed this inhibition, indicating that Bmp1 binds directly to miR-128-3p via the hypothetical MRE in the "ceRNA" regulatory network.

We used bioinformatics analysis to identify a potential Phf6 binding site on miR-128-3p, suggesting that Phf6 is targeted by miR-128-3p. To verify our bioinformatics analysis, qRT-PCR and western blot were used, showing that IEC-6 cells transfected with miR-128-3p mimic downregulated PHF6 expression, while transfection with the miR-128-3p inhibitor increased PHF6 expression. To determine whether miR-128-3p binds directly to Phf6, luciferase reporter gene analysis was carried out. MiR128-3p inhibited luciferase activity of the vector containing the Phf6 sequence. The putative mutation of the target site of miR-128-3p reversed this inhibitory effect, indicating that, in this "ceRNA" regulatory network, miR128-3p binds directly to Phf6 through the putative MRE. All these data indicate that miR-128-3p targets the Phf6 gene. To further verify whether Bmp1 regulates the process of proliferation and migration of IEC- 6 cells by regulating the expression of PHF6 through the sponge miR$128-3 p$, we carried out remedial experiments. The results 


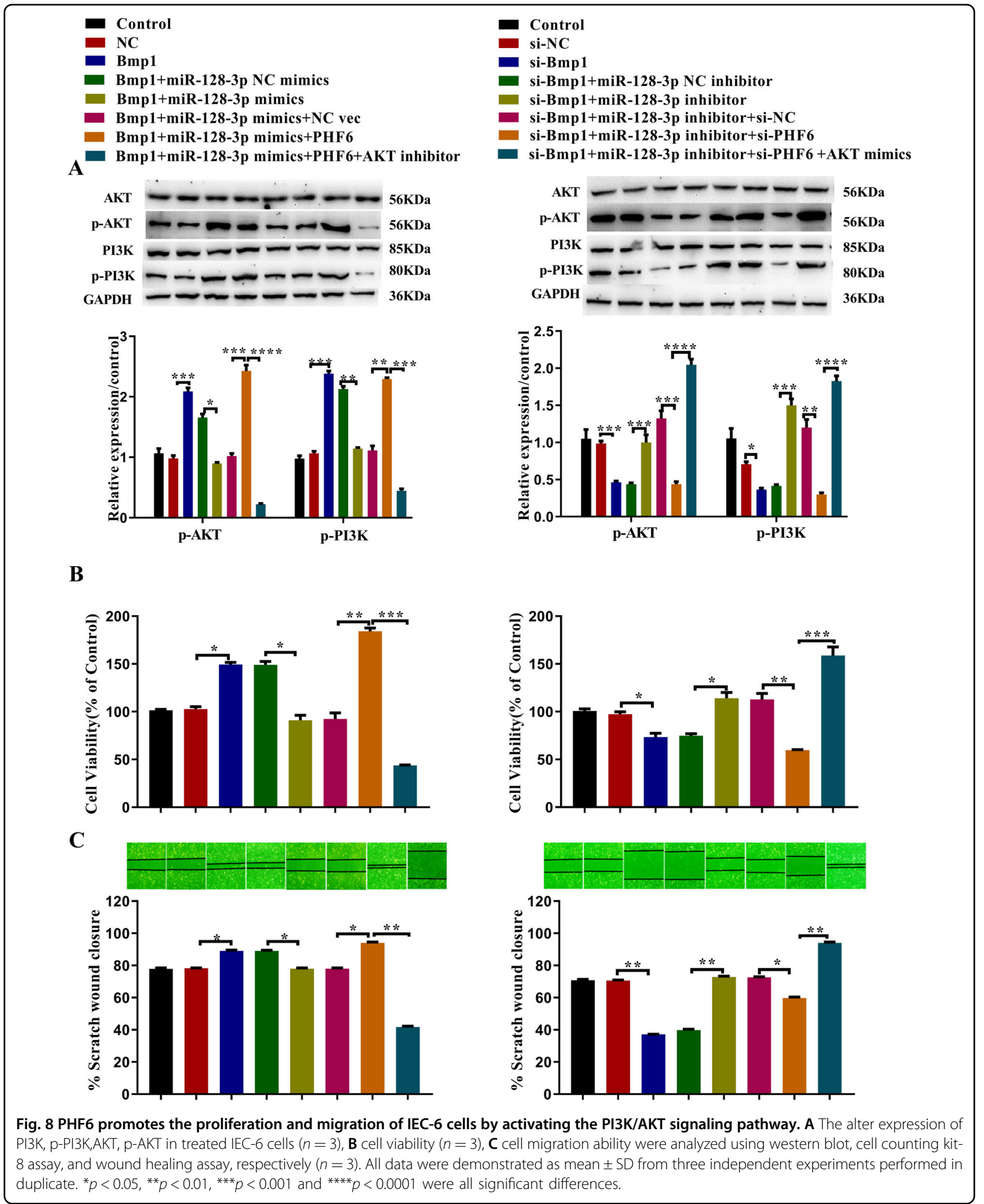


showed that the increased expression of miR-128-3p reverses Bmpl's promotion of cell proliferation and migration, and upregulation of PHF6 reversed the inhibitory effect of miR-128-3p on the function of Bmp1. The PI3K/AKT signaling pathway plays an important role in cell regulation such as cell proliferation and migration ${ }^{26}$. Therefore, the interaction between PI3K/AKT pathway and Bmp1 was evaluated. We found that overexpression of Bmp1 could activate thePI3K/AKT signaling pathway, upregulation of miR-128-3p reversed the effect of Bmp1 on the pathway, and upregulation of PHF6 reversed the inhibitory effect of miR-128-3p on the pathway. To sum up, these results show that Bmp1 promotes the epigenetic transcription of PHF6 by interacting with miR-128-3p, thus promoting the activation of the PI3K/AKT signaling pathway.

In conclusion, our data revealed that high expression of Bmp1 may promote the repair of damaged intestinal mucosa through ceRNA mediation and regulation of PHF6 expression by sponging miR-128-3p. The PI3K/ AKT signaling may be a downstream factor in mediating the impact of Bmp1 on injured intestinal mucosa(Fig S1). The Bmp1-miR-128-3p-PHF6-PI3K/AKT axis may prove a promising approach for the diagnosis and treatment of damaged intestinal mucosa.

\section{Materials and methods}

\section{Experimental animals, burn model, tissue, and blood processing}

Healthy, clean, adult, C57BL/6J mice $(20-30 \mathrm{~g} ; n=50)$ were used in this study. This work was approved by the Animal Ethics Committee of Xuzhou Medical University, China on September 15, 2016. Mice were raised adaptively for one week under conditions of constant temperature and ventilation and were fasted with free water availability for $12 \mathrm{~h}$ before the experiment. The mice were anesthetized by intraperitoneal injection of $1 \mathrm{~g} / \mathrm{L}$ pentobarbital sodium $(40 \mathrm{mg} / \mathrm{kg})$. The fur on the back was removed with scissors and hair removal cream, exposing $30 \%$ of the body. After the bare skin was soaked in water at $100^{\circ} \mathrm{C}$ for $8 \mathrm{~s}, 50 \mathrm{~mL} / \mathrm{kg}$ of lactic acid was injected intraperitoneally, and the mice were given anti-shock treatment. This treatment had previously been shown to cause fullthickness burns. Mice were randomly divided into three groups: control group (C, $n=10)$, burn group (B, $n=10)$, and burn + Bmp1 group $(n=10)$. Bmp1 adenovirus (Shanghai Hanheng Bio-Technology, China) was administered to mice in the burn + Bmp1 group $(10 \mu \mathrm{l} / \mathrm{g}), 1 \mathrm{~h}$ before severe burns, by intragastric injection. Blood and intestinal samples were collected at designated time points after burns. Blood samples were taken, centrifuged at $4 .{ }^{\circ} \mathrm{C}(12,000 \times g, 20 \mathrm{~min})$ to prepare serum, and enzyme-linked immunosorbent assay (ELISA) was used to detect lipopolysaccharide (LPS), interleukin-6 (IL-6), and tumor necrosis factor- $\alpha$ (TNF- $\alpha)$. A portion of each intestinal tissue specimen was stored at $-80^{\circ} \mathrm{C}$ for western blotting and real-time PCR quantification, and the remaining specimens were fixed in $10 \%$ formalin solution for hematoxylin-eosin (H\&E) staining.

\section{ELISA}

The ELISA kit for detecting LPS, IL-6, and TNF- $\alpha$ in mouse serum was purchased from Sigma Biotechnology Company (St Louis, MI, USA) and used according to the manufacturer's instructions.

\section{Western blot analysis}

The cells were lysed with RIPA lysis buffer and the total protein concentration was measured with a Protein Assay Kit (Beyotime, China). Separate equal amounts of protein were electrophoresed on SDS-PAGE and transferred to PVDF membranes (Amersham, USA). The membrane was blocked with 5\% BSA at room temperature for $2 \mathrm{~h}$ and incubated with the primary antibody overnight. The primary antibodies for PHF6, PI3K, p-PI3K, p-AKT, and AKT were obtained from Abcam (Cambridge, MAUSA) and used at 1:800 dilution. MMP9, E-cadherin, and ZO-1 antibodies were purchased from Proteintech (Rosemont, IL, USA) and used at 1:10,000 dilution. Anti-GAPDH (BioWorld, Dublin, OH, USA) at 1:10,000 dilution was used as the loading control. The membrane was washed three times with Tris-buffered saline containing 0.05\% Tween 20 (TBST) for 5 min each time. Membranes were then incubated with secondary antibody (1:10000, Proteintech, Rosemont, IL, USA) at room temperature for $1 \mathrm{~h}$ and washed with TBST for $30 \mathrm{~min}$. Finally, Odyssey infrared imaging software Image Studio Version 5.2 (LiCor Biotechnology, Bad Homburg, Germany) was used to record the images. The western blot analysis used Image J software for quantitative analysis.

\section{Intestinal mucosa morphology}

The mice were killed, the intestines were removed, cut along the longitudinal axis, rinsed with isotonic saline, and flattened. We observed the mucosal damage under a magnifying glass. The view of each sample was divided into five sections and each section was scored separately. These five scores were then added to provide a final damage score for the sample. The scoring method followed a previous study ${ }^{27}$ and the scoring system is presented in Table S1.

\section{H\&E staining}

The intestinal tissue was preserved with $10 \%$ formalin solution for pathological sectioning (thickness $3 \mu \mathrm{m}$ ). We used the method described by Perez-Chanona et al. to assess the state of intestinal injury ${ }^{28}$. The scoring system is presented in Table S2. 


\section{Cell culture}

The rat intestinal epithelial cell (IEC-6) line was obtained from the Shanghai Institute of Biological Sciences (Shanghai, China) and was cultured in RPMI-1640 with $10 \%$ fetal bovine serum (FBS) (Zhejiang Tianhang Biotechnology Co., Ltd., Zhejiang, China) at $37^{\circ} \mathrm{C}$ and $5 \%$ carbon dioxide. The media were supplemented with $100 \mathrm{U}$ penicillin and $100 \mu \mathrm{g}$ streptomycin per $\mathrm{ml}$. The human intestinal epithelial cells (HIEC-6) used in this study were purchased from ATCC (Manassas, VA, USA). HIEC-6 cells were cultured at $37^{\circ} \mathrm{C}$ and $5 \% \mathrm{CO}_{2}$ in highglucose DMEM containing 5\% FBS, $10 \mathrm{mM}$ HEPES, $4 \mathrm{mM}$ glutamine, $50 \mathrm{U} / \mathrm{ml}$ penicillin, $50 \mathrm{mg} / \mathrm{ml}$ streptomycin, $5 \mathrm{mg} / \mathrm{ml}$ epidermal growth factor, and $100 \mathrm{ml} / \mathrm{ml}$ insulin, transferrin, and selenium.

\section{Adenovirus infection}

The recombinant adenovirus and scrambled control (NC) were obtained from the Shanghai Hanheng Company. The GeneBank accession number of Bmp1 is NR_033241.1. Cells were transfected by adenovirus (MOI $=200$ ) exposure in $8 \mathrm{~mL}$ RPMI-1640 supplemented with $10 \%$ FBS for $24 \mathrm{~h}$. The infected cells were cultured in a conventional medium, and the expression level of Bmp1 was detected.

\section{Transfection of miRNA mimic/ inhibitor}

MiRNA mimic/inhibitor were obtained from Shenggong Company (Shanghai, China). After culturing to $90 \%$ confluence, 6-well plates were inoculated with $2 \times 10^{5}$ cells per well. The miRNA mimic/ miRNA NC mimic $(50 \mathrm{nmol} / \mathrm{L})$, or miRNA inhibitor/miRNA NC inhibitor $(20 \mathrm{nmol} / \mathrm{L})$ were transfected into the cells using $\mathrm{X}$ tremeGENE siRNA Transfection Reagent (Roche, Switzerland). After incubation for $48 \mathrm{~h}$, the cell proteins and the total RNA were extracted from the cells, and expression of the endogenous miR-128-3p was detected using qRT-PCR.

\section{siRNA transfection}

Cells were transfected with siRNA reagents using XtremeGENE siRNA Transfection Reagent (Roche, Switzerland) according to the manufacturer's protocol. The pooled siRNAs for knocking down the expression of Bmp1(20nmol/L), PHF6 $(20 \mathrm{nmol} / \mathrm{L})$, and the negative control (NC) were purchased from Hanheng Company (Shanghai, China). After transfection for $72 \mathrm{~h}$, the expression of Bmp1 and PHF6 mRNA was assessed by qRT-PCR.

\section{Transfection of PHF6 overexpression plasmid}

The PHF6 overexpression plasmid was obtained from Hanheng Company (Shanghai, China). The IEC-6 cells were seeded in 6 -well plates $\left(2 \times 10^{5}\right.$ cells per well). The
PHF6 plasmid $(50 \mathrm{nmol} / \mathrm{L})$ was transfected into the cells using LipoFiter 3 (Hanheng, Shanghai, China). After incubation for $72 \mathrm{~h}$, the expression of PHF6 was measured using qRT-PCR and Western blot.

\section{AKT mimic (SC79) and inhibitor (ly294002) transfection AKT mimic transfection}

Five milligrams of AKT mimic (Beyotime company, Nanjing, China) were added to $1.37 \mathrm{ml}$ DMSO, to prepare $10 \mathrm{mM}$ solution. One day before transfection, $2 \times 10^{5}$ cells were inoculated into each well of a 6-well plate and the culture medium replaced with fresh cell culture medium without phenol red. After $24 \mathrm{~h}$ of culture, the previously configured solution $(8 \mu \mathrm{g} / \mathrm{ml})$ was added and cultured overnight. The follow-up experiment was carried out $20 \mathrm{~h}$ later.

\section{AKT inhibitor transfection}

The day before transfection, $2 \times 10^{5}$ cells per well were inoculated in 6-well plates and grown until 50\%-70\% confluent. After changing to fresh cell culture medium, the final concentration of the AKT inhibitor $(\mathrm{APE} \times \mathrm{BIO}$, USA), was $10 \mu \mathrm{M}$. After gently mixing, the follow-up experiment was carried out after incubation for $24 \mathrm{~h}$.

\section{Quantitative real-time PCR analysis of IncRNA, miRNA, and mRNA levels}

Real-time PCR was used to quantify Bmp1, miR-128-3p, and PHF6. Total RNA of tissues and cells was extracted by Trizol (Invitrogen, CA, USA). Reverse transcription was performed using miRcute miRNA first-strand cDNA synthesis kit (Tiangen Biotechnology, Beijing, China) according to the manufacturer's instructions. The internal references were GAPDH or U6. Real-time fluorescent quantitative PCR was performed using the LightCycler 480 system (Roche, Basel, Switzerland) and Fast Universal SYBR Green Master Mix (Roche). In the melting curve analysis, there is only one peak per PCR product. The abundance of mRNA was calculated by the $2^{-\Delta \Delta \mathrm{Ct}}$ method, considering the gene-specific efficiency, and normalized to the above average expression index.

The primers used in this experiment are listed in Table S3.

\section{Cell counting kit-8 (CCK-8) assay}

After transfection for $24 \mathrm{~h}$, the cells were digested, collected, and seeded into 96-well plates at $2 \times 10^{3}$ per well. Each group was set up with six replicates. The CCK8 method (Dojindo, Kumamoto, Japan) was used to measure the cell survival rate at different time points. Ten microliters of CCK- 8 solution was added to each well $2 \mathrm{~h}$ before the assay and incubated at $37^{\circ} \mathrm{C}$ for $1 \mathrm{~h}$. The absorbance of each well at $450 \mathrm{~nm}$ was measured with a 
microplate reader (Thermo Fisher Scientific, Waltham, MA, USA).

\section{Colony formation assay}

After $24 \mathrm{~h}$ transfection, cells were plated onto a 6-well plate at a density of 500 cells per well and cultured for consecutive 10 days until colony formation. Then the cells were washed with PBS, fixed in methanol for $30 \mathrm{~min}$ and stained with $0.1 \%$ crystal violet dye. The colonies $(>50$ cells per colony) were observed and counted under a light microscope. All assays were performed in triplicate.

\section{Wound-healing assay}

The cells were seeded in 6-well plates at a density of $2 \times$ $10^{5}$ cells $/ \mathrm{ml}$ and incubated until they reached $90-100 \%$ confluence. Subsequently, an artificial wound was carefully made using a sterile P-10 pipette tip. The cells were thereafter treated differently for an additional 24,48 , or $72 \mathrm{~h}$ in serum-free medium. The rate of cell migration into the wound area was examined and photographed. The degree of wound contraction in a single group was quantified by the percentage of the area of cells that had migrated into the wound relative to the total area of the wound. Experiments were repeated three times.

\section{Immunofluorescence assay}

Immunofluorescence assay was used to detect Ecadherin expression in cells. After different treatments, the cells were cultured on glass coverslips in 6-well plates and fixed with $4 \%$ paraformaldehyde for $10 \mathrm{~min}$ at room temperature. The cells were then permeabilized with $0.1 \%$ Triton X-100 for $10 \mathrm{~min}$ on ice. The cells were blocked with goat serum (DCS/BioGenex, Hamburg, Germany) for $60 \mathrm{~min}$ at room temperature and then incubated with the primary antibody (1:50, Proteintech) overnight at $4{ }^{\circ} \mathrm{C}$. The cells were then incubated with FITC-tagged secondary antibodies (1:100, Huaan Company, China) for $60 \mathrm{~min}$ at room temperature. Subsequently, cells were washed and nuclei were counterstained with DAPI (Beyotime Company, China). Images were captured using a laser scanning confocal microscope (Olympus, Japan).

\section{Dual-luciferase reporter assay}

The bioinformatic websites miRWalk and TargetScan were used to predict the binding sites between Bmp1 and miR-128-3p and between miR-128-3p and PHF6. The dualluciferase reporter gene experiment was performed to verify whether miR-128-3p was the target gene of Bmp1 and Phf6 was the target gene of miR-128-3p. Bmp1 transcripts (NR_033241.1) and Phf6 3'-UTR with or without mutations within miR-128-3p binding sites were cloned into the psiCHECK-2 luciferase reporter vector (Promega, Madison, WI, USA). The miR-128-3p mimic or negative control (NC) were cotransfected with the reporter plasmid for $48 \mathrm{~h}$. The determination of the relative luciferase activity was done by the dual luciferase reporter gene detection system (Promega, Madison, WI, USA).

\section{Statistical analysis}

Statistical analysis was performed using SPSS 19.0 software (IBM Corp., Armonk, NY, USA). Data are expressed as mean \pm standard deviation (SD). Student's $t$-test or one-way analysis of variance (ANOVA) was used to evaluate the differences between groups. $P<0.05$ was considered statistically significant.

\section{Acknowledgements}

We would like to acknowledge the reviewers for their helpful comments on this paper.

\section{Author contributions}

M.M.Z., Y.Q.D., W.W.Z., B.Z., H.Y., J.Q.L., and Y.S. contributed to the conception and design of the study. M.M.Z., Y.Q.D., W.W.Z., B.Z., H.Y., J.Q.L., and Y.S.

contributed to the acquisition, analysis, and/or interpretation of data. M.M.Z., Y. Q.D., W.W.Z., B.Z., H.Y., J.Q.L., and Y.S. contributed to drafting and revising the manuscript, and P.Z., Q.W.C., H.T., and H.S. provided final approval of the version to be submitted.

\section{Funding}

This work was supported by the National Natural Science Foundation of China [grant number 81772082], the Natural Science Foundation of Jiangsu Province [grant number BK20151150], and the Natural Science Foundation of Xuzhou [grant number KC20085].

\section{Data availability}

The data sets used and/or analyzed during the current study are available from the corresponding author on reasonable request.

\section{Ethics statement}

This study was approved by the Animal Ethics Committee of Xuzhou Medical University.

\section{Conflict of interest}

The authors declare no competing interests.

\section{Publisher's note}

Springer Nature remains neutral with regard to jurisdictional claims in published maps and institutional affiliations.

Supplementary information The online version contains supplementary material available at https://doi.org/10.1038/s41419-021-03879-2.

Received: 4 February 2021 Revised: 26 May 2021 Accepted: 27 May 2021 Published online: 09 June 2021

\footnotetext{
References

1. Deitch, E. A. Multiple organ failure. Pathophysiology and potential future therapy. Ann. Surg. 216, 117-134 (1992).

2. Chen, X. Small RNAs and their roles in plant development. Annu Rev. Cell Dev. Biol. 25, 21-44 (2009).

3. Ulitsky, l., Shkumatava, A., Jan, C. H., Sive, H. \& Bartel, D. P. Conserved function of lincRNAs in vertebrate embryonic development despite rapid sequence evolution. Cell 147, 1537-1550 (2011).

4. Evans, J. R., Feng, F. Y. \& Chinnaiyan, A. M. The bright side of dark matter: IncRNAs in cancer. J. Clin. Invest. 126, 2775-2782 (2016).
} 
5. Ge, Q., Dong, Y., Lin, G. \& Cao, Y. Long noncoding RNA antisense noncoding RNA in the INK4 locus correlates with risk, severity, inflammation and infliximab efficacy in Crohn's disease. Am. J. Med. Sci. 357, 134-142 (2019).

6. Geng, $\mathrm{H}$. et al. In inflamed intestinal tissues and epithelial cells, interleukin 22 signaling increases expression of $\mathrm{H} 19$ long noncoding RNA, which promotes mucosal regeneration. Gastroenterology 155, 144-155 (2018).

7. Shuwen, H., Qing, Z., Yan, Z. \& Xi, Y. Competitive endogenous RNA in colorectal cancer: a systematic review. Gene 645, 157-162 (2018).

8. lqbal, M. A., Arora, S., Prakasam, G., Calin, G. A. \& Syed, M. A. MicroRNA in lung cancer: role, mechanisms, pathways and therapeutic relevance. Mol. Asp. Med 70, 3-20 (2019)

9. Wang, J. Y. et al. Potential regulatory role of IncRNA-miRNA-mRNA axis in osteosarcoma. Biomed. Pharmacother. 121, 109627 (2020).

10. Franzoni, E., et al. miR-128 regulates neuronal migration, outgrowth and intrinsic excitability via the intellectual disability gene Phf6. Elife 4, e04263 (2015).

11. Lower, K. M. et al. Mutations in PHF6 are associated with Borjeson-ForssmanLehmann syndrome. Nat. Genet. 32, 661-665 (2002).

12. Van Vlierberghe, P. et al. PHF6 mutations in T-cell acute lymphoblastic leukemia. Nat. Genet 42, 338-342 (2010).

13. $\mathrm{Yu}, \mathrm{Q}$. et al. Downregulation of PHF6 Inhibits cell proliferation and migration in hepatocellular carcinoma. Cancer Biother Radiopharm. 34, 245-251 (2019).

14. Zhang, C. et al. The X-linked intellectual disability protein PHF6 associates with the PAF1 complex and regulates neuronal migration in the mammalian brain. Neuron 78, 986-993 (2013).

15. Lou, G. et al. Direct targeting sperm-associated antigen 9 by miR-141 influences hepatocellular carcinoma cell growth and metastasis via JNK pathway. J. Exp. Clin. Cancer Res. 35, 14 (2016).

16. Shukal, D. et al. Dichloroacetate prevents TGFbeta-induced epithelialmesenchymal transition of retinal pigment epithelial cells. Exp. Eye Res. 197, 108072 (2020)
17. Spessotto, $P$. et al. Hyaluronan-CD44 interaction hampers migration of osteoclast-like cells by down-regulating MMP-9. J. Cell Biol. 158, 1133-1144 (2002).

18. Huo, L. et al. Cdc42-dependent formation of the ZO-1/MRCKbeta complex at the leading edge controls cell migration. EMBO J. 30, 665-678 (2011).

19. Zhang, $L$. et al. Valproic acid inhibits prostate cancer cell migration by upregulating E-cadherin expression. Pharmazie 66, 614-618 (2011).

20. Ge, Y., Sun, Y. \& Chen, J. IGF-II is regulated by microRNA-125b in skeletal myogenesis. J. Cell Biol. 192, 69-81 (2011).

21. Cannon, A. R. et al. Effects of mesalamine treatment on gut barrier integrity after burn injury. J. Burn Care Res. 37, 283-292 (2016).

22. Earley, Z. M. et al. Burn injury alters the intestinal microbiome and increases gut permeability and bacterial translocation. PLOS ONE 10, e0129996 (2015).

23. Yuan, N. et al. Integrative analysis of IncRNAs and miRNAs with coding RNAs associated with ceRNA crosstalk network in triple negative breast cancer. Onco Targets Ther. 10, 5883-5897 (2017).

24. Wei, C. et al. Long noncoding RNA LnC-SEMT modulates IGF2 expression by sponging miR-125b to promote sheep muscle development and growth. Cell Physiol. Biochem. 49, 447-462 (2018).

25. Lan, X. et al. Long noncoding RNA Inc-HC regulates PPARgamma-Mediated hepatic lipid metabolism through miR-130b-3p. Mol. Ther. Nucleic Acids $\mathbf{1 8}$ 954-965 (2019).

26. Mendez-Pertuz, M. et al. Modulation of telomere protection by the PI3K/AKT pathway. Nat. Commun. 8, 1278 (2017).

27. Millar, A. D. et al. Evaluating the antioxidant potential of new treatments for inflammatory bowel disease using a rat model of colitis. Gut 39, 407-415 (1996).

28. Perez-Chanona, E., Muhlbauer, M. \& Jobin, C. The microbiota protects against ischemia/reperfusion-induced intestinal injury through nucleotide-binding oligomerization domain-containing protein 2 (NOD2) signaling. Am. J. Pathol. 184, 2965-2975 (2014). 